\title{
Friends, Foes, and Favorites: Relative Interactions Determine How Polymer Brushes Absorb Vapors of Binary Solvents
}

\author{
Leon A. Smook, Guido C. Ritsema van Eck, and Sissi de Beer*
}

Cite This: Macromolecules 2020, 53, 10898-10906

Read Online

ABSTRACT: Polymer brushes can absorb vapors from the surrounding atmosphere, which is relevant for many applications such as in sensing and separation technologies. In this article, we report on the absorption of binary mixtures of solvent vapors (A and B) with a thermodynamic mean-field model and with grandcanonical molecular dynamics simulations. Both methods show that the vapor with the strongest vapor-polymer interaction is favored and absorbs preferentially. In addition, the absorption of one vapor
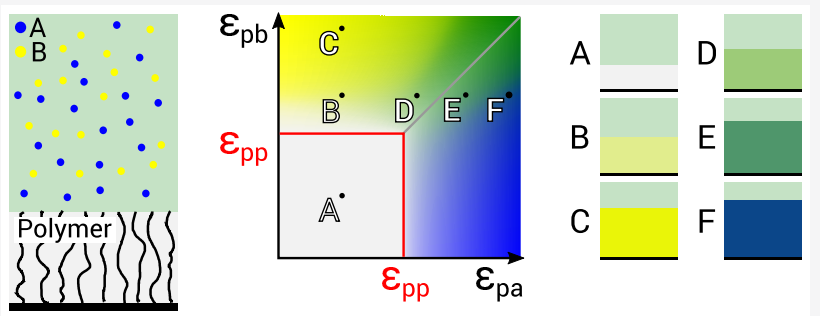
(A) influences the absorption of another (B). If the A-B interaction is stronger than the interaction between vapor $\mathrm{B}$ and the polymers, the presence of vapor $\mathrm{A}$ in the brush can aid the absorption of $\mathrm{B}$ : the vapors absorb collaboratively as friends. In contrast, if the A-polymer interaction is stronger than the B-polymer interaction and the brush has reached its maximum sorption capacity, the presence of A can reduce the absorption of B: the vapors absorb competitively as foes.

\section{INTRODUCTION}

The behavior of polymer brushes ${ }^{1,2}$ depends strongly on their interaction with the environment. The surroundings of such brushes alter their properties: ${ }^{3}$ in good solvents, brushmodified surfaces can reduce friction, ${ }^{4-7}$ provide antifouling properties, ${ }^{8}$ or introduce stimulus-responsive behavior, ${ }^{9}$ which can be employed in sensing applications, ${ }^{10,11}$ gating, ${ }^{12,13}$ or pick-up and place systems for nanoparticles. ${ }^{14}$ So far, brush properties have been mostly explored in liquids, whereas for many applications, for example, in vapor sensing, ${ }^{15,16}$ moisture harvesting, ${ }^{17,18}$ or gas separations, ${ }^{19-22}$ the brushes will be exposed to air. Brushes can swell in solvent vapors, ${ }^{23-27}$ and these vapor-swollen brushes resemble liquid-swollen brushes with respect to their solvent distribution and scaling behavior ${ }^{28}$ even though qualitative differences exist between both types of swelling.

Brush swelling by vapors depends on multiple system properties. In a previous study ${ }^{29}$ from our group, simulations showed that the interparticle interactions, partial vapor pressure, and grafting density all influence the absorption of single-component vapors in contact with brushes. It was found that absorption is described relatively well by using a simple mean field model based on the Flory-Huggins theory of solvation. $^{30,31}$ Additionally, the simulations revealed that vapors can also adsorb on top of the polymer brushes, depending on the polymer-solvent interactions. These studies of single-component vapor sorption provide the first necessary insights into understanding brushes in contact with vapors. However, in many practical applications (such as gas separation $^{19-22}$ and sensing $\left.{ }^{15,16}\right)$, brushes will be exposed to multicomponent gas mixtures. In fact, the component of interest might be present as a trace vapor in a gas mixture that already solvates the brush.

To understand the solvation of polymer brushes in contact with vapor mixtures, we study a Lennard-Jones gas mixture with a range of compositions in contact with a coarse-grained polymer brush model via grand-canonical molecular dynamics simulations. The results of these simulations agree to a remarkable extend with a box model based on the chemical equilibrium between the absorbed and free vapor. Both methods reveal that the presence of one component can change the sorption of another component, giving rise to competitive, collaborative, and preferential absorption.

Mean Field Box Model. A chemical system at equilibrium lacks particle fluxes since there is no gradient in chemical potential $(\mu)$. The absence of such gradients allows for setting up a model for polymer brushes to describe the vapor fractions in the brush. This sorption model requires a description of the chemical potential of the vapor everywhere in the system, which can be seen as two boxes-inside and outside the brush-with equal chemical potential for the solvent (see Figure 1). ${ }^{30}$

Received: September 29, 2020

Revised: November 17, 2020

Published: December 4, 2020 


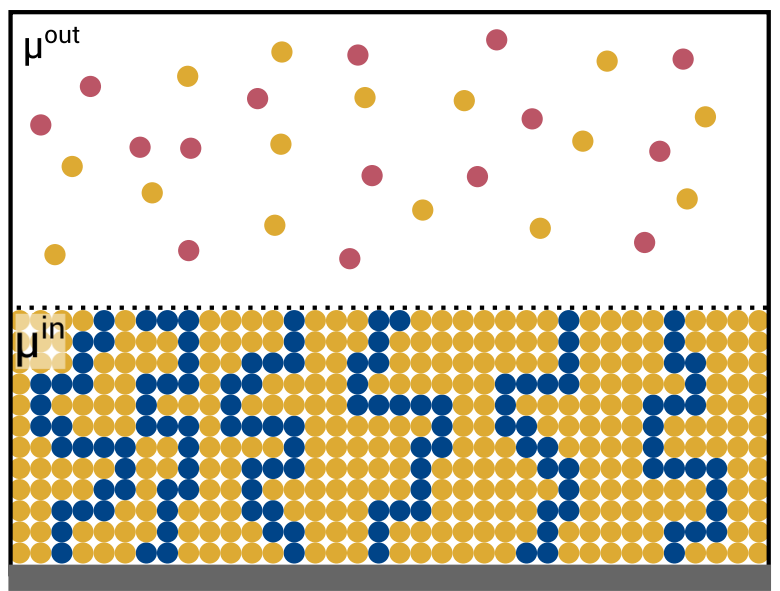

Figure 1. Schematic representation of the thermodynamic box model. Vapor particles inside and outside the brush are at chemical equilibrium $\left(\mu_{i}^{\text {out }}=\mu_{i}^{\text {in }}\right)$. The chemical potential inside the brush is influenced by mixing entropy, binary interactions, and chain stretching. The chemical potential outside the brush follows from the vapor pressure relative to the saturation vapor pressure.

Under an ideal gas assumption, the chemical potential of the vapor outside the brush can be expressed relative to a pure fluid at reference conditions as

$$
\mu^{\text {out }}=\ln \left(\frac{p}{p_{\text {sat }}}\right)
$$

where $\mu^{\text {out }}$ is in units of $k_{\mathrm{B}} T$ and $p$ and $p_{\text {sat }}$ are the vapor and saturation pressure, respectively.

The chemical potential of the vapor inside the brush follows from differentiation with respect to the number of solvent particles of the free energy. This free energy depends on contributions from molecular interactions, mixing, and chain stretching. The interaction and mixing contributions are described using the Flory-Huggins theory ${ }^{32}$ for the free energy of mixing with a slight modification-we follow Birshtein and Lyatskaya ${ }^{30}$ and exclude the translational entropy contribution for the grafted chains. The stretching contribution follows from the expression for the free energy of stretching of a polymer chain. For a brush in contact with a two-component vapor, these contributions can be summarized in an expression for the free energy $F^{\text {in }}$ (in $k_{\mathrm{B}} T$ ) associated with a single polymer in the brush ${ }^{30}$

$$
\begin{aligned}
F^{\text {in }} & =\underbrace{N_{\mathrm{A}} \ln \phi_{\mathrm{A}}+N_{\mathrm{B}} \ln \phi_{\mathrm{B}}}_{\text {entropy of mixing }} \\
& +\underbrace{\phi_{\mathrm{P}}\left(\chi_{\mathrm{A}} N_{\mathrm{A}}+\chi_{\mathrm{B}} N_{\mathrm{B}}\right)+N_{\mathrm{A}} \phi_{\mathrm{B}} \chi_{\mathrm{AB}}}_{\text {binary interactions }}+\underbrace{\frac{3 H^{2}}{2 D_{\mathrm{P}}}}_{\text {stretching }}
\end{aligned}
$$

where $H$ indicates the brush height (nondimensionalized with respect to the monomer size $a), D_{\mathrm{P}}$ is the number of monomers per chain, and $\phi_{i}$ and $N_{i}$ are respectively the volume fraction and number of particles of type $i$. The interactions between different particle types follow from the FloryHuggins $\chi$ parameters where the indices refer to the interacting pair; interaction with the polymer is assumed if only one particle type is mentioned. Note that the brush height can be expressed as a function of the number of monomers per chain, the grafted area per chain, and the volume fraction of polymer such that $H=\frac{D_{\mathrm{P}}}{s \phi_{\mathrm{p}}}$, where $s$ is the grafted area per chain (nondimensionalized by $a^{2}$ ).

The chemical potentials inside and outside the brush are equal at equilibrium, giving a set of two equations. This set is complemented with a volumetric constraint-the sum of all number/volume fractions equals one-which completes the model as

$$
\left\{\begin{aligned}
\ln \left(\frac{p_{\mathrm{A}}}{p_{\mathrm{A}, \mathrm{sat}}}\right)= & \ln \phi_{\mathrm{A}}+\phi_{\mathrm{P}}+\left(\chi_{\mathrm{AB}} \phi_{\mathrm{B}}+\chi_{\mathrm{A}} \phi_{\mathrm{P}}\right)\left(\phi_{\mathrm{B}}+\phi_{\mathrm{P}}\right)-\chi_{\mathrm{B}} \phi_{\mathrm{B}} \phi_{\mathrm{P}} \\
& +\frac{3}{\phi_{\mathrm{P}} \mathrm{s}^{2}} \\
\ln \left(\frac{p_{\mathrm{B}}}{p_{\mathrm{B}, \mathrm{sat}}}\right)= & \ln \phi_{\mathrm{B}}+\phi_{\mathrm{P}}+\left(\chi_{\mathrm{AB}} \phi_{\mathrm{A}}+\chi_{\mathrm{B}} \phi_{\mathrm{P}}\right)\left(\phi_{\mathrm{A}}+\phi_{\mathrm{P}}\right)-\chi_{\mathrm{A}} \phi_{\mathrm{A}} \phi_{\mathrm{P}} \\
& +\frac{3}{\phi_{\mathrm{P}} s^{2}} \\
\phi_{\mathrm{A}}+\phi_{\mathrm{B}}+ & \phi_{\mathrm{P}}=1
\end{aligned}\right.
$$

where the first two equations balance the chemical potential in the vapor (LHS) and brush (RHS) phase and the third equation imposes the volumetric constraint.

The applicability of the model is limited by its simplicity and implicit assumptions. We assume a grafting density larger than the critical grafting density - the distance between two anchor points is smaller than twice the radius of gyration of a chain in a poor solvent. Moreover, we assume that the brush composition is homogeneous throughout the brush. However, inhomogeneities perpendicular to the grafting surface are not expected for collapsed brushes since density profiles of collapsed brushes often show a sharp brush/gas interface ${ }^{28}$ as well as a nearly height-independent density ${ }^{24,29}$ (see also Figure S1). Large inhomogeneities parallel to the surface are prevented by the anchoring of the polymer chains; the minimum and maximum distances between chains due to this end attachment provide natural limits to the brush composition. In fact, we hypothesize that systems that do not reach an energetic minimum within these limits would form a vertical partitioning, ${ }^{33}$ modifying the brush interface instead of the brush composition.

Despite these limitations, the box model accounts for the system properties mentioned in the Introduction which have been shown to influence sorption behavior: interparticle interactions (or equivalently brush and vapor chemistry) are taken into account in the Flory-Huggins interaction parameters, brush properties are taken into account in the grafted area per chain, and vapor properties are taken into account by the vapor and saturation pressure.

Linking Thermodynamic Box Model to Molecular Dynamics Simulations. The system of eqs 3 describes vapor sorption in polymer brushes from a thermodynamic perspective. Yet, linking this thermodynamic description to molecular dynamics simulations is challenging due to assumptions underlying these equations and its variables. While some of these parameters (the grafting density, saturation pressure, and vapor pressure) follow from the input for the simulation or are easily assessed by complementary molecular dynamics simulations, a challenge rises when mapping these simulations onto an interaction parameter. 
The interaction parameter between species $\alpha$ and $\beta$ is defined as an exchange energy by

$$
\chi_{\alpha \beta}=\frac{z}{k_{\mathrm{B}} T}\left(\frac{1}{2}\left(\epsilon_{\alpha \alpha}+\epsilon_{\beta \beta}\right)-\epsilon_{\alpha \beta}\right)=\frac{z}{k_{\mathrm{B}} T} W_{\alpha \beta}
$$

where $z$ is the coordination number of the Flory-Huggins lattice, $k_{\mathrm{B}} T$ is the thermal energy, $\epsilon$ is the interaction energy of the particle pair in the subscript, and $W_{\alpha \beta}$ is the exchange energy between species $\alpha$ and $\beta$. Because $W_{\alpha \beta}$ follows from the simulation parameters, this expression maps the MD simulations onto the interaction parameter with a scaling factor that includes the temperature and the coordination number.

Using a scaling factor to directly map interaction energies onto interaction parameters oversimplifies the system since it includes several assumptions. First, we assume a constant coordination number for all systems, even though this number may be influenced by system parameters such as interaction strengths-the liquid structure may change for different systems. Second, these systems include a gas phase, whereas the interaction parameter is based on a liquid phase exchange energy, which may introduce a systematic error in the mapping.

Keeping in mind these limitations, the mapping according to eq 4 connects the model to the input and output of the simulations directly. The simulations, however, do not directly provide the coordination number. This parameter follows from fitting the model to each simulation data point. In a first approximation, we assume the coordination number to be constant for all systems. However, this is not necessarily true and a variation in this number is allowed if and only if this variation can be supported by changes in the liquid structure in the system.

Molecular Dynamics Setup. To investigate vapor sorption, we performed molecular dynamics (MD) simulations of brushes of Kremer-Grest chains ${ }^{34}$ in contact with a Lennard-Jones gas mixture of vapors A and B, as depicted in Figure 2. This gas mixture was kept at a constant chemical potential by using a Monte Carlo approach-vapor particles were inserted or deleted according to the Metropolis criterion on every 10000th time step during the MD time integration. This time integration was performed by using the LAMMPS ${ }^{35}$ software package using the rRESPA integration scheme ${ }^{36}$ with two hierarchical levels where each large time step contains two small time steps of $0.0075 \tau .^{a}$ Bonded interactions are computed in the innermost loop, and pair forces are computed in the outer loop.

To simulate brush solvation, a brush of monodisperse Kremer-Grest chains consisting of 120 beads with a grafting density of $0.133 \sigma^{-2}\left(s=7.52 \sigma^{2}\right)$ was used; these parameters ensure a system in a brush regime even in its collapsed state (see the Supporting Information). The chains were end-grafted in a simulation box $\left(30 \times 30 \times 130 \sigma^{3}\right)$ with fixed boundary conditions on the upper and lower wall (parallel to the grafting surface) and periodic boundary conditions in all other directions (perpendicular to the grafting surface). The chain anchors were excluded from time integration so that the chains remain end-grafted during the simulation. The fixed boundary condition was enforced by a "mathematical wall" with a harmonic potential with a spring constant of $100 \epsilon \sigma^{-2}$ and a cutoff of $1 \sigma$ to prevent particles leaving the simulation box. A $20 \sigma$ region below the upper box wall was used for grand-

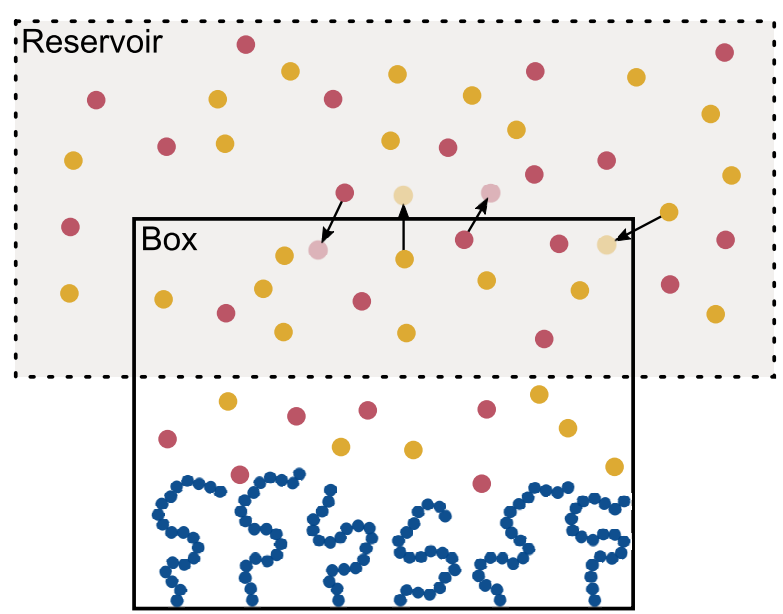

Figure 2. Graphical representation of the GCMC-MD simulation box. The top part of the simulation box (indicated with solid borders) is in equilibrium with an implicit atmosphere (indicated with dashed borders). This equilibrium is maintained by a Metropolis algorithm that determines the success of insertion/deletion of a particle from the surrounding atmosphere into the simulation box as indicated by the arrows. Only the contents of the simulation box are explicitly simulated in the MD procedure between GCMC sweeps.

canonical particle insertion/deletion of the Lennard-Jones gas with a set vapor pressure. ${ }^{37,38}$ This region was located below the cutoff of the wall potential such that this potential does not affect the GCMC procedure.

Particle interactions result from well-defined interaction potentials. A potential shifted Lennard-Jones potential $\left(U_{\mathrm{LJ}, \mathrm{PS}}\right)$ governs interactions between nonbonded particles as a function of the distance between them $(r)$, which is described by

$$
\begin{aligned}
& U_{\mathrm{LJ}}(r)=4 \mathrm{\epsilon}\left(\left(\frac{\sigma}{r}\right)^{12}-\left(\frac{\sigma}{r}\right)^{6}\right) \\
& U_{\mathrm{LJ}, \mathrm{PS}}(r)= \begin{cases}U_{\mathrm{LJ}}(r)-U_{\mathrm{LJ}}\left(r_{\mathrm{c}}\right) & \text { for } r \leq r_{\mathrm{c}} \\
0 & \text { for } r>r_{\mathrm{c}}\end{cases}
\end{aligned}
$$

where the nonshifted interaction strength $(\epsilon)$ at the equilibrium distance $r_{\mathrm{m}}$ is used to define the interaction between particles. To reduce the computational load, a cutoff distance $r_{\mathrm{c}}$ at $2.5 \sigma$ is used for the interaction potential. Note that these potentials make that all pair interactions in this work are attractive.

To investigate the effect of the relative interaction strength of the different vapors, we varied the interaction energy of each vapor-polymer pair between $0.2 \epsilon$ and $1.4 \epsilon$ with a constant vapor and polymer self-interaction. The polymer selfinteraction $\left(\epsilon_{\mathrm{PP}}\right)$ was set at 0.6 ; the vapor self-interaction $\left(\epsilon_{\mathrm{AA}}, \epsilon_{\mathrm{BB}}\right)$ (as well as the vapor cross-interaction $\epsilon_{\mathrm{AB}}$ ) was set at 1.0. Simulations were performed at a low and high vapor pressure relative to the saturation pressure of each vapor component $\left(p_{\text {low }}=0.05 p_{\text {sat }} ; p_{\text {high }}=0.34 p_{\text {sat }}\right)$ with a one-to-one ratio between both vapors. In another set of simulations, the vapor ratio was varied, and several combinations of vaporpolymer interactions were used, namely, $\epsilon_{\mathrm{PA}}=0.8 \epsilon$ and $\epsilon_{\mathrm{PB}}=$ $[0.4,0.6,0.75] \epsilon$. Here, the combined vapor pressure of both components was kept constant (i.e., $p_{\mathrm{A}}+p_{\mathrm{B}}=$ constant). 
The potential describing the interaction between bonded particles $\left(U_{\text {bond }}\right)$ is a combination of a finite extensible nonlinear elastic (FENE) potential and a Weeks-ChandlerAndersen (WCA) potential, such that

$$
\begin{aligned}
& U_{\mathrm{FENE}}(r)=-0.5 K R_{0}^{2} \ln \left(1-\left(\frac{r}{R_{0}}\right)^{2}\right) \\
& U_{\mathrm{WCA}}(r)= \begin{cases}U_{\mathrm{LJ}}(r)+\epsilon & \text { for } r \leq 2^{1 / 6} \sigma \\
0 & \text { for } r>2^{1 / 6} \sigma\end{cases} \\
& U_{\text {bond }}(r)=U_{\mathrm{FENE}}(r)+U_{\mathrm{WCA}}(r)
\end{aligned}
$$

where $K$ is $30 \epsilon \sigma^{-2}, R_{0}$ is $1.5 \sigma, \epsilon$ is 1 , and $\sigma$ is 1 . This choice of parameters prevents unphysical behavior and bond crossing. ${ }^{34}$

To ensure vapor-liquid coexistence, the simulations were performed at a temperature of $0.85 \epsilon k_{\mathrm{B}}$. The value for $k_{\mathrm{B}}$ is set to 1 - the use of reduced LJ units allows us to scale energy and temperature. We controlled the temperature using a chain of three Nosé-Hoover thermostats with a damping constant of $0.15 \tau$ (where $\tau$ is the reduced unit of time), which allows a proper sampling of the canonical ensemble. ${ }^{39}$

To reduce simulation artifacts due to nonrepresentative starting configurations, we simulate vapor solvation using a two-step process: equilibration and production. The equilibration consists of an energy minimization of a fully stretched brush to a collapsed state. This collapsed state is then used in the grand-canonical molecular dynamic simulations-a Lennard-Jones vapor is introduced via the GCMC region by 1000 particle insertion/deletion attempts every 10000 time steps in the MD simulation. The simulation is continued until the brush composition stabilizes (typically after $10^{4} \tau-10^{5} \tau$ ), after which 500000 time steps are performed to capture the statistics of the system.

During the data collection time steps, we collect density profiles (see Figure $3^{40}$ ) perpendicular to the grafting plane. From these density profiles, we define the brush height as the inflection point of this profile. Next, the brush profiles are integrated over the height to calculate the composition of the brush; the density profile corresponding to a particle type gives the number of particles sorbed in the brush. By calculating the

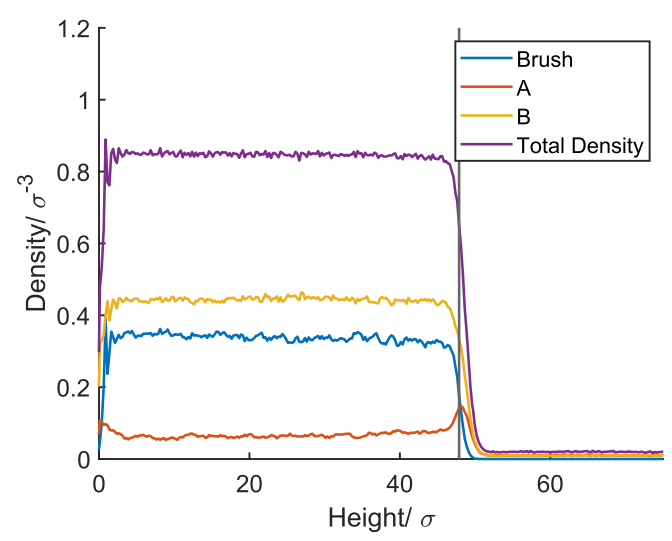

Figure 3. Representative density profile showing brush height (gray vertical line), density of polymer (blue), density of vapors A and B (red and yellow), and the total density inside the brush (purple). The profile shown is for $\epsilon_{\mathrm{PA}}=1.0$ and $\epsilon_{\mathrm{PB}}=1.4$ at a relative vapor pressure of $34 \%$ for each component. height of the brush based on the inflection point, the vapor particles that adsorb on top of the brush are not included in the sorption. A selection of representative density profiles is presented in the Supporting Information.

\section{RESULTS AND DISCUSSION}

The absorption of binary solvent vapors in polymer brushes is investigated using grand-canonical molecular dynamics simulations of Kremer-Grest brushes exposed to a binary LennardJones vapor consisting of components $\mathrm{A}$ and $\mathrm{B}$. The absorption of component $\mathrm{A}$ is evaluated with respect to the presence of the second vapor $\mathrm{B}$. For binary mixtures with a one-to-one ratio of $A$ and $B$, the interaction strength of both vapors with the brush was varied to sample a variety of relative interactions. We remind the reader that all pair interactions in this work are attractive. This sampling is performed at high $\left(p=0.34 p_{\text {sat }}\right)$ and low $\left(p=0.05 p_{\text {sat }}\right)$ relative vapor pressure for each component. Finally, the absorption of vapor mixtures with different ratios is investigated for a selection of relative interactions.

Absorption is evaluated based on the density profiles of the polymer and solvents. Figure 3 shows such a profile for a oneto-one vapor mixture at high relative pressure with $\epsilon_{\mathrm{PA}}=1.0$ and $\epsilon_{\mathrm{PB}}=1.4$. This figure displays all typical features of vapor swollen density profiles. The density inside the brush is independent of the height coordinate, indicating that a box model is a fair representation of the system. Moreover, the relative number densities of vapors A and B follow logically from their interactions; $\mathrm{B}$ has a stronger interaction with the brush than $\mathrm{A}\left(\epsilon_{\mathrm{PB}}=1.4\right.$ vs $\left.\epsilon_{\mathrm{PA}}=1.0\right)$ and hence absorbs more. Finally, we note that the density of vapor A displays a maximum at the brush interface. This is the result of the effective interaction between vapor $\mathrm{A}$ and the local brush/ vapor environment. At the interface, the density of the brush decreases quickly, reducing its influence on the sorption of vapors in the region. At the same point, the density of vapor $B$ is still significant, and B has a stronger interaction with $A$ than the brush, leading to a coadsorption of the vapors onto the brush. We note that most of the adsorbed vapor is present at heights above our definition of the brush height; hence, the observed maximum will not significantly affect the accuracy of the box model.

Competitive and Collaborative Absorption. For a single-component vapor, the absorption of a vapor depends on the strength of the interaction between this vapor and the brush. $^{29}$ For a component in a binary vapor, this trend also holds. Figure $4 \mathrm{a}$ shows the fraction of $\mathrm{A}$ in the brush as a function of the interaction strength between the brush and $\mathrm{A}$ $\left(W_{\mathrm{PA}}\right)$ for a binary vapor with a 1:1 ratio between $\mathrm{A}$ and $\mathrm{B}$ at a relative vapor pressure of $34 \%$. We note that the curves of $W_{\mathrm{PB}}$ $=0.4$ and $W_{\mathrm{PB}}=0.6$ overlap. We observe that a stronger interaction between the brush and A, which means a lower value of $W_{\mathrm{PA}}$, results in a higher absorption of this component for all interaction strengths of the vapor $B$ with the brush $\left(W_{\mathrm{PB}}\right)$. Absorption is driven by the negative interaction energy of solvent inclusion in the brush and opposed by interactionindependent entropic effects (vapor localization and brush stretching). Hence, a stronger interaction between a component and brush leads to more absorption of this component. Nevertheless, this increase is affected by the absorption of the second component $\mathrm{B}$ for $W_{\mathrm{PB}}<0$. This effect is most pronounced for $W_{\mathrm{PB}}=-0.2$ and $W_{\mathrm{PA}}=-0.2$ where the absorption is reduced by $26 \%$ with respect to $W_{\mathrm{PB}}=0.6$ and 

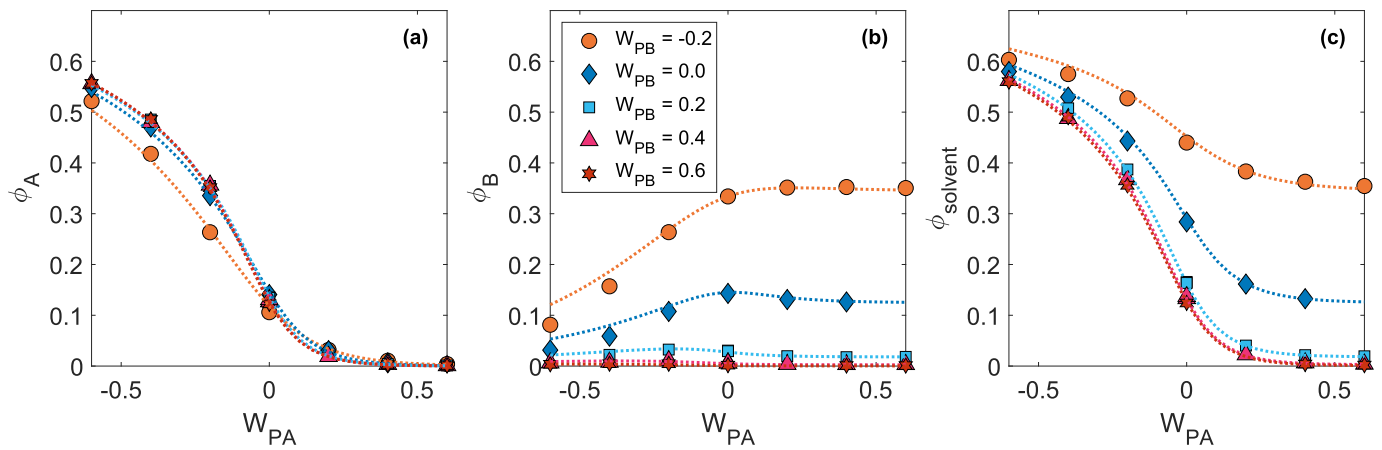

Figure 4. Component fractions of a Kremer-Grest brush in contact with a Lennard-Jones vapor consisting of two different particle types (A, B) in a one-to-one ratio at a relative vapor pressure of $34 \pm 1 \%$. The dotted line are model values for the mapping $\chi=9.51 \mathrm{~W}$ (based on a fit over all data points in this work). (a) Fraction of component A in the brush. (b) Fraction of component B in the brush. (c) Total solvent fraction (A + B) in the brush.

the same value for $W_{\mathrm{PA}}(-0.2)$. For these conditions, the interactions of both components with the brush are equal, which leads to competitive absorption between vapors $\mathrm{A}$ and $\mathrm{B}$ : the absorption of vapor $\mathrm{B}$ reduces the absorption of $\mathrm{A}$.

Simultaneously, the absorption of component B is affected by the absorption of component A. This effect is presented in Figure $4 \mathrm{~b}$ with the absorption of component $\mathrm{B}$ versus the interaction strength between the brush and component $\mathrm{A}$ $\left(W_{\mathrm{PA}}\right)$. This figure reveals two regimes depending on the interaction between $\mathrm{B}$ and the brush. For weak B-brush interactions $\left(W_{\mathrm{PB}} \geq 0.2\right)$, the effect of component $\mathrm{A}$ on the absorption of component $\mathrm{B}$ is negligible. However, if $\mathrm{B}$ interacts significantly with the brush $\left(W_{\mathrm{PB}}<0.2\right)$, we observe a strong effect on the absorption of this component when the interaction between the brush and component $\mathrm{A}$ increases (i.e., when the interaction parameter between $\mathrm{A}$ and the brush decreases). In other words, when component $A$ interacts more strongly with the brush than component $\mathrm{B}$, the relative amount of $B$ in the brush decreases. This effect follows from both free energy and volumetric constraints. Both vapors release energy when they absorb, and thus both vapors "want" to absorb. However, the entropic penalty due to chain stretching depends not on the type of particle absorbed, but only on the number of particles. This provides a limit to the number of absorbed particles, and hence, components A and B compete for this available space. However, note that this available space is a function of the interaction strength between the vapor components and the brush, and thus the total sorption can vary.

Figure $4 \mathrm{~b}$ also indicates that the absorption of $\mathrm{B}$ goes through a maximum at $W_{\mathrm{PA}}=0$ for $W_{\mathrm{PB}}=0$ and at $W_{\mathrm{PA}}=-0.2$ for $W_{\mathrm{PB}}=0.2$. Here absorption is collaborative in the sense that the presence of $\mathrm{A}$ aids the absorption of $\mathrm{B}$. This can be understood considering the polymer-solvent interactions relative to the interaction between $\mathrm{A}$ and $\mathrm{B}$. In the vapor phase, components A and B do not interact with other solvent particles under ideal gas assumptions. In the brush, these vapor particles do interact with each other and the polymer. Hence, the energy that is released upon absorption is not only a result of interaction with the brush but also a result of interaction with other solvent particles. Considering that the solventsolvent interaction $\left(\epsilon_{\mathrm{AB}}=1.0\right)$ in our simulations is stronger than the brush-solvent interaction for the selected points $\left(\left[\epsilon_{\mathrm{PA}}=0.8, \epsilon_{\mathrm{PB}}=0.8\right]\right.$, and $\left.\left[\epsilon_{\mathrm{PA}}=1.0, \epsilon_{\mathrm{PB}}=0.6\right]\right)$, the absorption of vapor in the brush lowers the chemical potential of the other component, resulting in a collaborative absorption.
We note that this effect differs from the cosolvency effect in binary solvents with respect to two key aspects. First, cosolvency ${ }^{41}$ is limited to mixtures of poor solvents, whereas collaborative absorption is not. Second, cosolvency results from entropic contributions of the liquid phase, and collaborative absorption results from an effective enthalpic interaction between the brush and the vapor.

The total absorption of vapor in the brush (so A and B) increases when a single component has a stronger interaction with the brush. Figure $4 \mathrm{c}$ shows the total absorption of vapor $(\mathrm{A}+\mathrm{B})$ versus the interaction between the brush and component $\mathrm{A}\left(W_{\mathrm{PA}}\right)$. For all $W_{\mathrm{PB}}$, a stronger interaction between brush and component $\mathrm{A}$ results in a higher solvent fraction in the brush. However, the increase in the solvent fraction differs depending on the interaction of the brush and component $\mathrm{B}$. To illustrate, for $W_{\mathrm{PB}}=0.6$ the solvent fraction ranges from 0 to 0.56 whereas for $W_{\mathrm{PB}}=-0.2$ it only ranges from 0.35 to 0.60 . This effect results from the absorption of component $\mathrm{B}$, since a lower $W_{\mathrm{PB}}$ indicates a stronger interaction between the brush and $\mathrm{B}$. For lower values of $W_{\mathrm{PB}}(<0.2)$, some $\mathrm{B}$ will absorb for all values of $W_{\mathrm{PA}}$, introducing a background absorption that is relatively unaffected by the value $W_{\text {PA }}$ affecting the total absorption.

The aforementioned effects are also present at lower vapor pressures. A stronger interaction between a vapor and the brush results in a higher absorption of that vapor, even though the amount of vapor absorbed is less than at higher vapor pressures (Figure S2a). This lower absorption also affects the competitive absorption. Though this effect remains present, its magnitude is significantly reduced as a result of this overall lower absorption (Figure S2b). Moreover, the total vapor absorption increases with increasing interaction between brush and vapor (Figure S2c). Hence, relative vapor pressure does not qualitatively affect the absorption behavior; it merely has a quantitative effect. For instance, for $W_{\mathrm{PB}}=-0.2$ and $W_{\mathrm{PA}}=0.0$ the fraction of $\mathrm{B}$ in the brush is reduced from 0.33 at high vapor pressures to 0.10 at low vapor pressures. Energetically this results from the entropic loss when a vapor absorbs. This loss is more significant for more dilute vapors, which have more entropic freedom.

Preferential Absorption. The competitive character of the absorption of binary vapors indicates a preference for the absorption of the stronger interacting component. Such a preference for the absorption of one component over the other is called preferential absorption, and it results in a discrepancy between the A-B ratio in the vapor $\left(\Phi_{\mathrm{A}}\right)$ and the brush phase 
$\left(\phi_{\mathrm{A}} / \phi_{\text {solvent }}\right)$. Figure 5 shows this vapor versus brush phase ratio at $W_{\mathrm{PA}}=0$ and for $W_{\mathrm{PB}}=0.05,0.2$, and 0.4 . In the

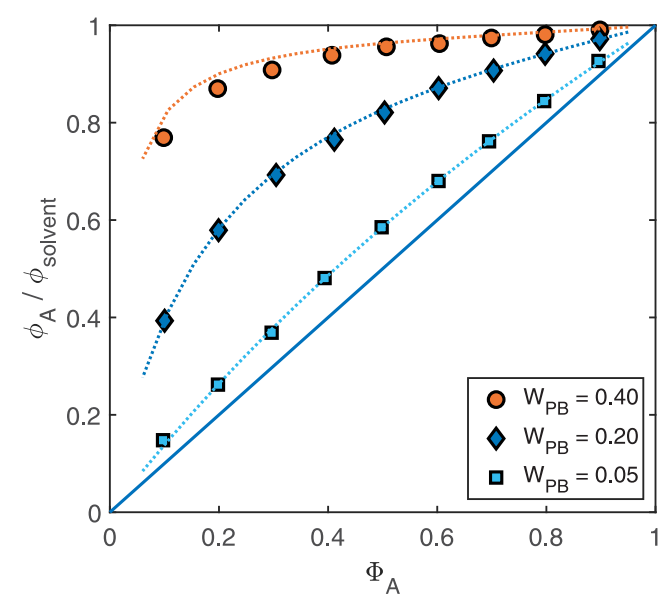

Figure 5. Fraction of solvent $\mathrm{A}$ in the brush $\left(\phi_{\mathrm{A}} / \phi_{\text {solvent }}\right)$ as a function of the fraction of solvent $A$ in the vapor phase $\left(\Phi_{\mathrm{A}}\right)$ for $W_{\mathrm{PA}}=0.0$ and $W_{\mathrm{PB}}=0.05,0.20$, and 0.40 . The dotted line are model values for the mapping $\chi=9.51 \mathrm{~W}$ (based on a fit over all data points in this work). The component with the stronger interaction with the polymer (solvent A here) absorbs more than the weaker component (solvent B). This effect is stronger with a larger difference in affinity.

absence of preferential absorption, vapor and brush phase ratios should be equal. However, the figure shows a discrepancy from this ideality in the form of an excess of component $\mathrm{A}$, the component with the stronger absorption. This effect increases for stronger differences between the interaction of both vapors; however, even for small differences $(\Delta W=0.05)$ this effect is clearly observed. Thus, the component with the stronger interaction with the brush absorbs preferentially.

Comparison to the Thermodynamic Box Model. Competitive, collaborative, and preferential absorption follow not only from molecular dynamics simulations but also from the thermodynamic box model. Even though the model describes the system in a simplified manner, it shows a decent fit to the data. In a first-order approximation, we retrieve the proportionality factor between the exchange energy $\left(W_{\alpha \beta}\right)$ and the interaction parameter $\left(\chi_{\alpha \beta}\right)$ by fitting our model to all data points. This fit gives $\chi_{\alpha \beta}=(9.51 \pm 0.02) W_{\alpha \beta}$ [95\% CI, $N=$ 97]. ${ }^{b}$ Model values with this mapping are presented in Figures 4 and 5 and Figure S2 as dotted lines. In all figures, the model describes the data fairly well, and competitive, collaborative and preferential absorption also follow from the model.

Despite this qualitative agreement, we observe several quantitative differences under significant preferential and competitive absorption. For example, in Figure $4 b$, compare the theoretical value (dotted line) with the simulation data (markers) for $W_{\mathrm{PB}} \leq 0.0$ and $W_{\mathrm{PA}} \leq-0.4$. For these values, the model overestimates the absorption of $\mathrm{B}$. This discrepancy follows from the assumption that the coordination number is equal for all systems evaluated. To test this assumption, we simulate solvent imbibed melts of Kremer-Grest polymer chains with the same parameters as the chains in the brush. The polymer and solvent fractions in the melt are chosen so that they resemble the fractions observed in the brush for a specific set of interaction parameters. This imbibed melt is then simulated under constant pressure and temperature and the radial distribution function within these melts is retrieved. This method attempts to recreate the bulk of the brush phase in the brush simulations (see the Supporting Information for simulation details).

Figure 6 shows the radial distribution functions of selected melt compositions. Under the assumption of a constant

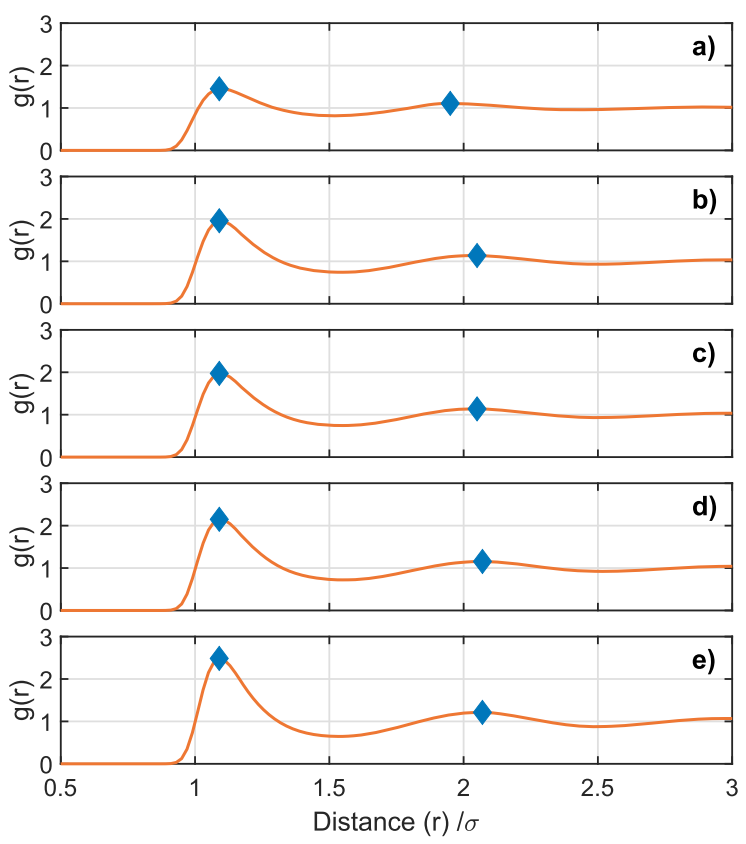

Figure 6. Radial distribution functions of Lennard-Jones solvent imbibed Kremer-Grest melts. From top to bottom, the average interaction strength between the polymer and the solvent mixture increases. (a) $W_{\mathrm{PA}}=0.6, W_{\mathrm{PB}}=0.6$. (b) $W_{\mathrm{PA}}=-0.2, W_{\mathrm{PB}}=0.6$. (c) $W_{\mathrm{PA}}=-0.2, W_{\mathrm{PB}}=0.0$. (d) $W_{\mathrm{PA}}=-0.2, W_{\mathrm{PB}}=-0.2$. (e) $W_{\mathrm{PA}}=-0.2$, $W_{\mathrm{PB}}=-0.6$.

coordination number, the radial distribution function should remain unchanged for different compositions. However, in the figure one can observe significant changes in the region between $0.5 \sigma$ and $3 \sigma$. The maximum of the first peak increases when the particle at the highest concentration interacts more strongly. To illustrate, the first maximum of the distribution function increases from 1.97 in Figure $6 \mathrm{c}$ for a theta and good solvent mixture to 2.48 in Figure $6 \mathrm{e}$ for a good and very good solvent mixture-an increase of $26 \%$. This increase indicates that particles with a stronger interaction pack more efficiently around other particles, leading to a higher coordination number. When we increase the coordination number by a similar factor, we find a proportionality constant of 11.97 and an improved match between the model and simulations for the individual solvent components (Figure S3). Despite the improved fit for the individual components, the model still overestimates the solvent content in the brush. Hence, the change in liquid structure can only partially account for the discrepancy between the methods. The overestimation at strong interactions results from the assumption of a Gaussian brush. For these strong interactions the brush swells to up to $40 \%$ of the contour length of an individual chain; at such extensions the Gaussian model underestimates the entropic loss of the brush, leading to an overestimation of the amount of sorbed solvent.

The discrepancy between the model and molecular dynamics simulations does not invalidate the model as both 
represent a simplified representation of "real" experimental systems. Both methods include assumptions about the nature of interactions and ideal behavior of vapors. Even though the methods model "real" systems in their own way, both point to similar behavior: competitive, collaborative, and preferential absorption. This is a strong indication that these effects are not merely an artifact of the methods used.

\section{DISCUSSION}

Preferential, collaborative, and competitive absorption appear to be enthalpy-driven phenomena. The free energy released upon absorption needs to counteract the entropic phenomena that disfavor absorption. As a result, at low vapor concentrations (the vapor is in a high entropy state), more interaction energy is required for absorption. Similarly, at high vapor concentrations, the vapor absorbs more readily. When two vapors are exposed to a polymer brush, these considerations hold. The vapor that best counteracts the entropic desorption force will absorb more. This leads to preferential absorption of this component. Additionally, the entropic desorption force limits the space available for absorption, and this space has to be shared between all absorbing components, which leads to competitive absorption.

We have performed simulations at a grafting density of $0.133 \sigma^{-2}$, which is well within the brush regime for the chain length used. For these conditions, we observe a good qualitative match between our model and simulations; both methods show collaborative, competitive, and preferential absorption. If we vary the grafting density in our model, we do not observe large qualitative differences in competitive (Figure S4) and preferential (Figure S5) absorption. However, a qualitative difference is observed for collaborative absorption. A higher grafting density leads to less collaborative absorption: from an increase due to collaborative absorption of over $17 \%$ for a grafting density of $0.1 \sigma^{-2}$ to only $2 \%$ for $0.5 \sigma^{-2}$ compared to a noninteracting second vapor component. This is the result of the entropic stretching penalty due to chain stretching; for higher grafting densities, less solvent absorbs since the polymer chains are more strongly stretched in the dry state compared to a brush with a lower grafting density. Hence, the entropic penalty of stretching due to absorption is increased, leading to a lower absorption in general. Additionally, the higher volume fraction of polymer limits the number of solvent-solvent contacts in the swollen brush, reducing the driving force behind collaborative absorption. In short, this means that at low grafting densities (but still in the brush regime) collaborative, competitive, and preferential absorption are observed; at higher grafting densities, competitive and preferential absorption remain, but collaborative absorption is reduced or even absent.

Preferential absorption of binary mixtures has previously been observed in polymer brushes exposed to binary liquids. For instance, theoretical work indicates that brushes exposed to a binary mixture of good and bad solvents preferentially absorb the good solvent. ${ }^{42}$ Neutron scattering experiments demonstrated this effect in poly(dimethylsiloxane) (PDMS) brushes with the preferential solvation of dicholoromethane (good solvent for PDMS) over methanol (poor solvent). ${ }^{43}$ In a similar experiment on poly(styrene) brushes, preferential solvation was observed in solvent mixtures of two poor solvents, a good and poor solvent, and two good solvents. ${ }^{44}$ Despite the similarities between preferential absorption in liquid and vapor solvated brushes, there is an important difference between both systems. Liquid solvated brushes are exposed to a mixture of solvents, whereas vapor solvated brushes are exposed to a gaseous medium. As a result, vapor solvated brushes are exposed to a mostly "poor solvent" medium, even when high concentrations of good solvent vapor are present. This effect results in a relatively sharp interface between the brush and vapor phase compared to a similar interface between a brush and liquid phase. ${ }^{28}$ This has important implications for the behavior of these brushes, since this collapsed ground state will require more energy to swell than a brush exposed to a good solvent.

A similar phenomenon, preferential adsorption (note the " $\mathrm{d}$ " instead of " $b$ " in adsorption), has been associated with cononsolvency ${ }^{45-47}$ in mixtures of good solvents. The preferential adsorption of one component onto the polymer can result in the formation of a "bridge" between two chains-they are connected via one solvent particle adsorbed on both chains, leading to a collapse of the brush (or reduction of the radius of gyration for a polymer in solution). A key difference between co-nonsolvency in mixtures of good solvents and (possible) cononsolvency in mixtures of solvent vapors is the surrounding medium. In good solvents, the polymer brush will be in a swollen state in either of the pure solvents. In good vapors, however, the polymer brush might swell but will still be collapsed compared to the good solvent case (compare the parabolic profiles in good solvents vs the step profiles in this work). Therefore, it is possible that "bridge" formation due to the adsorption of a better solvent vapor no longer leads to a further collapse of the brush. In fact, this better solvent might swell the brush even more and expel the poorer solvent by competitive absorption. We intend to explore the possibility of co-nonsolvation by vapors in future work.

Absorption effects are important to consider when designing sensors ${ }^{15}$ based on polymer brushes. For instance, preferential absorption due to differences in interaction strength makes that the "vapor" composition inside the brush is not identical with that outside of the brush. This is an important effect to take into account if one wants to sample the surrounding atmosphere. Note, however, that this effect is not limited to vapor sensing. Preferential absorption also exists in brushliquid mixtures ${ }^{48}$ (see also the discussion before) which may be an important consideration for surface-focused measurement techniques (for instance, attenuated total reflection infrared spectroscopy) in lab-on-a-chip applications.

The preferential effect is not perfectly selective and can thus be used to absorb a certain type of molecule from the surrounding atmosphere. One might even be able to tune the interaction such that only a specific subset of molecules absorbs. This customization and flexibility are promising in developing novel sensing technology based on nonspecific interactions. This nonspecificity shows promise for applications in gas-separation membranes ${ }^{49}$ where preferential, collaborative, and competitive effects can tune the relative permeability of the membrane for different gases.

\section{CONCLUSIONS}

To conclude, we have shown with a thermodynamic box model and with grand-canonical molecular dynamics simulations how vapor mixtures of binary solvents behave in contact with polymer brushes. The enthalpic interactions between the vapor components and the brush dominate the absorption. The component with the strongest interaction with the brush absorbs preferentially. This preferential absorption of the 
strongest interacting component leads to competitive absorption if multiple components absorb into the brush close to brush saturation. In contrast, the presence of primary solvent in the brush can also aid the absorption of the secondary solvent in the brush, resulting in collaborative absorption. This occurs when the interaction between the two solvents is stronger than the interaction between the secondary solvent and the brush. All these effects provide both challenges and opportunities in the design of new, nonspecific sensing and separation technologies.

\section{ASSOCIATED CONTENT}

\section{SI Supporting Information}

The Supporting Information is available free of charge at https://pubs.acs.org/doi/10.1021/acs.macromol.0c02228.

Density profile of a selection of polymer brushes; derivation of critical grafting density for collapsed brushes; details for imbibed melt simulations; vapor absorption for low vapor pressures; graphs of solvent fraction vs interaction parameter for different scaling factor; effect of grafting density on vapor absorption (PDF)

\section{AUTHOR INFORMATION}

\section{Corresponding Author}

Sissi de Beer - Sustainable Polymer Chemistry, University of Twente, 7500 AE Enschede, The Netherlands; 10 orcid.org/ 0000-0002-7208-6814; Phone: +31 (0)53 489 3170;

Email: s.j.a.debeer@utwente.nl

\section{Authors \\ Leon A. Smook - Sustainable Polymer Chemistry, University of Twente, 7500 AE Enschede, The Netherlands; (1) orcid.org/0000-0003-4176-8801 \\ Guido C. Ritsema van Eck - Sustainable Polymer Chemistry, University of Twente, 7500 AE Enschede, The Netherlands; (1) orcid.org/0000-0001-8697-6642}

Complete contact information is available at: https://pubs.acs.org/10.1021/acs.macromol.0c02228

\section{Notes}

The authors declare no competing financial interest.

\section{ACKNOWLEDGMENTS}

The authors thank L.B. Veldscholte for fruitful discussions and computational scripting support. NWO and SURFsara are acknowledged for HPC resources and support (project ref 45666). This work is part of the research program "Mechanics of Moist Brushes" with Project OCENW.KLEIN.020, which is financed by the Dutch Research Council (NWO).

\section{ADDITIONAL NOTES}

${ }^{a}$ Unless otherwise specified, reduced Lennard-Jones units are used in this work.

${ }^{b} \mathrm{CI}=$ confidence interval, $N=$ number of data points.

\section{REFERENCES}

(1) de Gennes, P. G. Conformations of Polymers Attached to an Interface. Macromolecules 1980, 13, 1069-1075.

(2) Milner, S. T. Polymer Brushes. Science 1991, 251, 905-914.
(3) Chen, W. L.; Cordero, R.; Tran, H.; Ober, C. K. 50th Anniversary Perspective: Polymer Brushes: Novel Surfaces for Future Materials. Macromolecules 2017, 50, 4089-4113.

(4) Klein, J.; Kumacheva, E.; Mahalu, D.; Perahia, D.; Fetters, L. J. Reduction of frictional forces between solid surfaces bearing polymer brushes. Nature 1994, 370, 634.

(5) Lee, S.; Spencer, N. D. Sweet, Hairy, Soft, and Slippery. Science 2008, 319, 575-577.

(6) de Beer, S.; Kutnyanszky, E.; Schön, P. M.; Vancso, G. J.; Müser, M. H. Solvent induced immiscibility of polymer brushes eliminates dissipation channels. Nat. Commun. 2014, 5, 3781.

(7) Singh, M. K.; Ilg, P.; Espinosa-Marzal, R. M.; Kröger, M.; Spencer, N. D. Polymer Brushes under Shear: Molecular Dynamics Simulations Compared to Experiments. Langmuir 2015, 31, 47984805.

(8) Maan, A. M.; Hofman, A. H.; de Vos, W. M.; Kamperman, M. Recent Developments and Practical Feasibility of Polymer-Based Antifouling Coatings. Adv. Funct. Mater. 2020, 30, 2000936.

(9) Chen, T.; Ferris, R.; Zhang, J.; Ducker, R.; Zauscher, S. Stimulusresponsive polymer brushes on surfaces: Transduction mechanisms and applications. Prog. Polym. Sci. 2010, 35, 94-112.

(10) Merlitz, H.; He, G.-L.; Wu, C.-X.; Sommer, J.-U. Nanoscale brushes: How to build a smart surface coating. Phys. Rev. Lett. 2009, 102, 115702.

(11) Klushin, L. I.; Skvortsov, A. M.; Polotsky, A. A.; Qi, S.; Schmid, F. Sharp and Fast: Sensors and Switches Based on Polymer Brushes with Adsorption-Active Minority Chains. Phys. Rev. Lett. 2014, 113, 068303.

(12) Coalson, R. D.; Eskandari Nasrabad, A.; Jasnow, D.; Zilman, A. A Polymer-Brush-Based Nanovalve Controlled by Nanoparticle Additives: Design Principles. J. Phys. Chem. B 2015, 119, 1185811866.

(13) Chen, G.; Das, S. Massively Enhanced Electroosmotic Transport in Nanochannels Grafted with End-Charged Polyelectrolyte Brushes. J. Phys. Chem. B 2017, 121, 3130-3141.

(14) Yu, Y.; Lopez de la Cruz, R. A.; Kieviet, B. D.; Gojzewski, H.; Pons, A.; Vancso, G. J.; de Beer, S. Pick up move and release of nanoparticles utilizing co-non-solvency of PNIPAM brushes. Nanoscale 2017, 9, 1670-1675.

(15) Li, L.; Li, J.; Lukehart, C. M. Graphitic carbon nanofiberpoly(acrylate) polymer brushes as gas sensors. Sens. Actuators, B 2008, 130, 783-788.

(16) McCaig, H. C.; Myers, E.; Lewis, N. S.; Roukes, M. L. Vapor Sensing Characteristics of Nanoelectromechanical Chemical Sensors Functionalized Using Surface-Initiated Polymerization. Nano Lett. 2014, 14, 3728-3732.

(17) Yang, H.; Zhu, H.; Hendrix, M. M. R. M.; Lousberg, N. J. H. G. M.; de With, G.; Esteves, A. C. C.; Xin, J. H. Temperature-Triggered Collection and Release of Water from Fogs by a Sponge-Like Cotton Fabric. Adv. Mater. 2013, 25, 1150-1154.

(18) Liu, X.; Li, Y.; Hu, J.; Jiao, J.; Li, J. Smart moisture management and thermoregulation properties of stimuli-responsive cotton modified with polymer brushes. RSC Adv. 2014, 4, 63691-63695.

(19) Balachandra, A. M.; Baker, G. L.; Bruening, M. L. Preparation of composite membranes by atom transfer radical polymerization initiated from a porous support. J. Membr. Sci. 2003, 227, 1-14.

(20) Bruening, M. L.; Dotzauer, D. M.; Jain, P.; Ouyang, L.; Baker, G. L. Creation of Functional Membranes Using Polyelectrolyte Multilayers and Polymer Brushes. Langmuir 2008, 24, 7663-7673.

(21) Pizzoccaro-Zilamy, M.-A.; Drobek, M.; Petit, E.; Totée, C.; Silly, G.; Guerrero, G.; Cowan, M. G.; Ayral, A.; Julbe, A. Initial Steps toward the Development of Grafted Ionic Liquid Membranes for the Selective Transport of $\mathrm{CO}_{2}$. Ind. Eng. Chem. Res. 2018, 57, 1602716040 .

(22) Aliyev, E.; Shishatskiy, S.; Abetz, C.; Lee, Y. J.; Neumann, S.; Emmler, T.; Filiz, V. SI-ATRP Polymer-Functionalized Graphene Oxide for Water Vapor Separation. Adv. Mater. Interfaces 2020, 7, 2000443. 
(23) Biesalski, M.; Rühe, J. Swelling of a polyelectrolyte brush in humid air. Langmuir 2000, 16, 1943-1950.

(24) Galvin, C. J.; Dimitriou, M. D.; Satija, S. K.; Genzer, J. Swelling of polyelectrolyte and polyzwitterion brushes by humid vapors. J. Am. Chem. Soc. 2014, 136, 12737-12745.

(25) Galvin, C. J.; Genzer, J. Swelling of Hydrophilic Polymer Brushes by Water and Alcohol Vapors. Macromolecules 2016, 49, 4316-4329.

(26) Gumerov, R. A.; Potemkin, I. I. Swelling of Planar Polymer Brushes in Solvent Vapors. Polym. Sci., Ser. C 2018, 60, 66-75.

(27) Brió Pérez, M.; Cirelli, M.; de Beer, S. Degrafting of Polymer Brushes by Exposure to Humid Air. ACS Applied Polymer Materials 2020, 2, 3039-3043.

(28) Sun, L.; Akgun, B.; Hu, R.; Browning, J. F.; Wu, D. T.; Foster, M. D. Scaling Behavior and Segment Concentration Profile of Densely Grafted Polymer Brushes Swollen in Vapor. Langmuir 2016, $32,5623-5628$.

(29) Ritsema van Eck, G. C.; Veldscholte, L. B.; Nijkamp, J. H. W. H.; de Beer, S. Sorption Characteristics of Polymer Brushes in Equilibrium with Solvent Vapors. Macromolecules 2020, 53, 84288437.

(30) Birshtein, T. M.; Lyatskaya, Y. V. Theory of the CollapseStretching Transition of a Polymer Brush in a Mixed Solvent. Macromolecules 1994, 27, 1256-1266.

(31) Orski, S. V.; Sheridan, R. J.; Chan, E. P.; Beers, K. L. Utilizing vapor swelling of surface-initiated polymer brushes to develop quantitative measurements of brush thermodynamics and grafting density. Polymer 2015, 72, 471-478.

(32) Flory, P. J. Principles of Polymer Chemistry; Cornell University Press: Ithaca, NY, 1953.

(33) Lyatskaya, Y.; Balazs, A. C. Phase separation of mixed solvents within polymer brushes. Macromolecules 1997, 30, 7588-7595.

(34) Kremer, K.; Grest, G. S. Dynamics of entangled linear polymer melts: A molecular-dynamics simulation. J. Chem. Phys. 1990, 92, 5057.

(35) Plimpton, S. Fast Parallel Algorithms for Short-Range Molecular Dynamics. J. Comput. Phys. 1995, 117, 1.

(36) Tuckerman, M.; Berne, B. J.; Martyna, G. J. Reversible multiple time scale molecular dynamics. J. Chem. Phys. 1992, 97, 1990-2001.

(37) Frenkel, D.; Smit, B. Understanding Molecular Simulation: From Algorithms to Applications, 2nd ed.; Academic Press: New York, 2002; Chapter 3, pp 23-61.

(38) Frenkel, D.; Smit, B. Understanding Molecular Simulation, 2nd ed.; Academic Press: New York, 2002; Chapter 8, pp 201-224.

(39) Braun, E.; Moosavi, S. M.; Smit, B. Anomalous Effects of Velocity Rescaling Algorithms: The Flying Ice Cube Effect Revisited. J. Chem. Theory Comput. 2018, 14, 5262-5272.

(40) Templates for the LAMMPS input files and initial configuration as well as the data underlying the figures of this article are available free of charge at DOI: 10.4121/13019537.

(41) Mukherji, D.; Marques, C. M.; Stuehn, T.; Kremer, K. Depleted depletion drives polymer swelling in poor solvent mixtures. Nat. Commun. 2017, 8, 1-7.

(42) Marko, J. F. Polymer Brush in Contact with a Mixture of Solvents. Macromolecules 1993, 26, 313-319.

(43) Auroy, P.; Auvray, L. Collapse-Stretching Transition for Polymer Brushes. Preferential Solvation. Macromolecules 1992, 25, 4134-4141.

(44) Auroy, P.; Auvray, L. Preferential Solvation in Poly(styrene) Brushes. Langmuir 1994, 10, 225-231.

(45) Mukherji, D.; Marques, C. M.; Kremer, K. Polymer collapse in miscible good solvents is a generic phenomenon driven by preferential adsorption. Nat. Commun. 2014, 5, 4882.

(46) Sommer, J. U. Adsorption-Attraction Model for Co-Nonsolvency in Polymer Brushes. Macromolecules 2017, 50, 2219-2228.

(47) Galuschko, A.; Sommer, J. U. Co-Nonsolvency Response of a Polymer Brush: A Molecular Dynamics Study. Macromolecules 2019, 52, 4120-4130.
(48) Cheng, J.; Vishnyakov, A.; Neimark, A. V. Morphological transformations in polymer brushes in binary mixtures: DPD study. Langmuir 2014, 30, 12932-12940.

(49) Keating, J. J.; Imbrogno, J.; Belfort, G. Polymer Brushes for Membrane Separations: A Review. ACS Appl. Mater. Interfaces 2016, $8,28383-28399$. 